\title{
Front End Schaltung zur Online Auswertung von EKG-Signalen
}

\author{
E. Ayari and R. Tielert \\ Technische Universität Kaiserslautern
}

\begin{abstract}
Zusammenfassung. Ein mobiles EKG-System zur Online Auswertung von EKG-Signalen wird dargestellt. Die Auswertung beruht auf ein energiesparendes Verfahren, das den Vorteil einer zulässigen Unterabtastung des Signals bietet und eine Interaktion zwischen der messenden Elektronik und dem funkgebundenen Auswertungsrechner ermöglicht. Diese Interaktion besteht darin, sowohl die Front End Schaltung im EKG-Sensor als auch den im ATmega8L eingebetteten A/D-Wandler vom Auswertungsrechner zu steuern und den Datenbedarf des Rechners dynamisch an die Erfordernisse des Analyseprogramms anzupassen. Das entwickelte EKG-System liefert erfolgreiche Charakterisierungen erfasster Elektrokardiogramme.
\end{abstract}

\begin{abstract}
A mobile ecg-system for an online analysis of electrocardiogram signals is presented. The analysis is based on an energy-saving procedure, which offers the advantage of an acceptable undersampling of the signal, and which allows an interaction between the measuring electronic and the radio-bound analysis-computer. In this interaction both the front-end circuit in the ecg-sensor and the A/D converter, which is embedded in the ATmega8L, are steered by the analysis computer. The data requirement of the computer is also dynamically adapted to the requirements of the analysis-program. The developed ecg-system supplies successful characterisations of measured electrocardiograms.
\end{abstract}

\section{Einleitung}

Bei einer typischen Langzeit-EKG-Untersuchung werden die Daten über einen längeren Zeitraum mobil gespeichert. Die Auswertung des aufgezeichneten EKGs erfolgt offline durch ein Analyseprogramm auf dem Rechner oder manuell durch einen Facharzt.

In dieser Arbeit wird eine mobile Messung des EKGSignals vorgestellt, bei der auf die Aufzeichnung verzich-

Correspondence to: E. Ayari

(zoghlami@eit.uni-kl.de) tet und eine kontinuierliche Analyse des aktuellen Signals ermöglicht wird. Beruhend auf einem energiesparenden Verfahren für eine kontinuierliche drahtlose EKG-Auswertung (Ayari-Tielert, 2005), das eine Interaktion zwischen der Sensor-Hardware und einem funkgebundenen Auswertungsrechner voraussetzt, wird ein energiearmer tragbarer Sensorgurt eingesetzt. Der Sensor übernimmt die Aufgaben der Vorverarbeitung des EKG-Signals bezüglich der Signalfilterung, der adaptiven Abtastung je nach Signalabschnitt und die Funkübertragung der Signaldaten zu einem „body area“ Netzwerk. Ein Mikroprozessor, der als zentraler Knoten am Körper getragen wird, übernimmt die kontinuierliche Auswertung.

Die beschränkte Rechenkapazität des Mikroprozessors setzt dem Auswertungsalgorithmus enge Grenzen. Je nach Anforderung werden verschiedene Merkmale des EKG- Signals extrahiert. Dies ist im einfachsten Fall die statistische Charakteristik der Herzfrequenz, die kontinuierlich durch Korrelationsanalyse von Puls zu Puls erfolgen kann. Weitere Merkmale des EKG werden durch Datenregression mit Musterschablonen ermittelt. Diese Analyse ist etwas aufwändiger und erfordert daher die Mittelung über mehrere Pulsfolgen. Die Ergebnisse sind in jedem Fall auf wenige charakteristische Daten beschränkt und können daher mit geringem Energieaufwand zum funkgebundenen Zentralknoten des Monitorsystems gesendet werden. Die Energieeffizienz des Systems wird durch die variablen Schlafperioden der Mess- und Sendeelektronik erreicht. Aufgrund der möglichen Miniaturisierung der Komponenten einschlielich der Batterien kann ein unauffällig getragenes mobiles Monitorsystem im Sinne von „Ambient Intelligence“ realisiert werden.

Passend zu diesen Anforderungen wird in dieser Arbeit die Implementierung einer programmierbaren Front End Schaltung in einer $0.18 \mu \mathrm{m}$ CMOS Technologie vorgestellt. Die Schaltung übernimmt die Vorverstärkung und adaptive Filterung des EKG- Signals und kooperiert mit einem Mikrocontroller, der auch die A/D-Wandlung und Steuerung der Funkübertragung übernimmt. Dank der Switched Capacitor

Published by Copernicus Publications on behalf of the URSI Landesausschuss in der Bundesrepublik Deutschland e.V. 


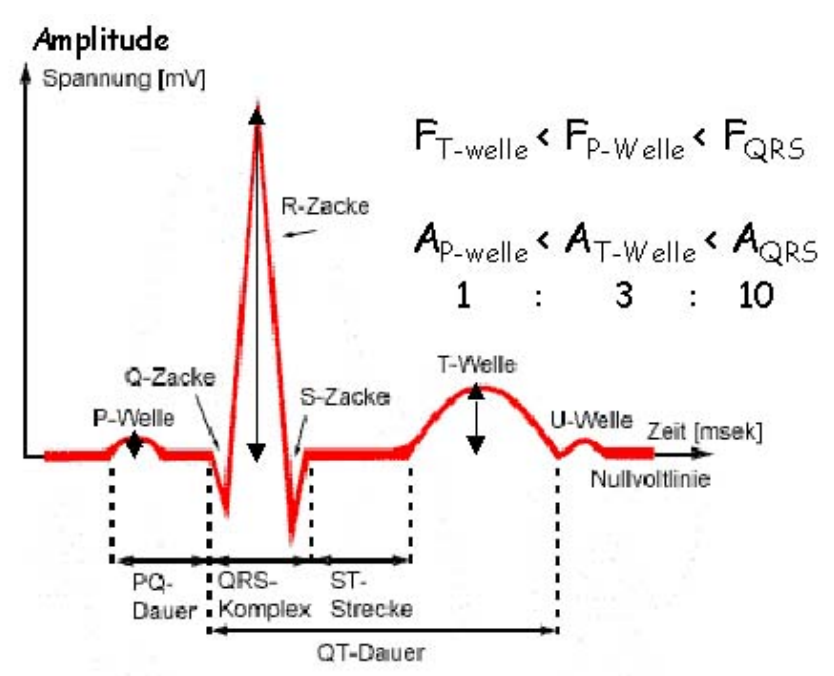

Abb. 1. Elektrokardiogramm.

Technik kann die Schaltung sehr variabel in Verstärkung und Grenzfrequenz durch den Mikrocontroller eingestellt werden. Gegenüber einer kontinuierlich aktiven Schaltung bietet die gewählte Technik den Vorteil geringer Toleranzempfindlichkeit. Die Schaltung mit aktiver Fläche von $420 \mu \mathrm{m} \times 320 \mu \mathrm{m}$ und einem Energieverbrauch von $600 \mu \mathrm{W}$ bei einer Versorgungsspannung von $3 \mathrm{~V}$ ist eine integrierte Komponente des hybrid aufgebauten Sensorboards.

\section{Charakterisierung des EKG-Signals}

Das Elektrokardiogramm(EKG) oder auch Herzstromkurve genannt ist die Registrierung der Summe der elektrischen Aktivitäten aller Herzmuskelfasern (Wikipedia, 2006). Das elektrische Strömungsfeld des Herzens breitet sich in das leitende Medium im Körper aus (Meyer-Waarden, 1984), so dass die elektrischen Potentialänderungen am Herzen an der Körperoberfläche abgegriffen werden können und in der Zeitachse aufgezeichnet werden. Es resultiert ein wiederkehrendes ziemlich gleichförmiges Bild der elektrischen Herzaktion, das EKG : siehe Abb. 1.

Ein typisches EKG-Signal besteht aus positiven und negativen Ausschlägen, die nach Einthoven als P, Q, R, S, T und U (Meyer-Waarden, 1984) bezeichnet werden. Sie bilden die 6 typischen Wellenformen eines EKG-Signals. Man unterscheidet im EKG zwischen Wellen oder Zacken, und Strecken oder Segmenten. Strecken sind die Abstände zwischen zwei Zacken. Die Charakterisierung des Signals verläuft durch die Beschreibung dieser Wellenformen auf der Zeit- und Amplituden-Achse, siehe Abb. 1.

Der Frequenzbereich des EKG-Signals liegt zwischen circa $0.05 \mathrm{~Hz}$ und $150 \mathrm{~Hz}$, wobei seine hochfrequenten Anteile im QRS-Komplex konzentriert sind.
Seine Amplitude ist sehr gering und liegt in der Regel im mV-Bereich wie bei jedem biologischen Signal. Die höchsten Amplituden sind beim QRS-Komplex registriert und sind circa 10-mal und 3-mal grösser als die P-Wellenund die T-Wellen-Amplituden jeweils.

\section{EKG-Sensor}

\subsection{Anforderungen an den drahtlosen EKG-Sensor}

Da Störsignale bis circa $20 \mathrm{mV}$ das niedrige Messsignal überlagern und es damit verfälschen können, ist eine differenzielle Messung erforderlich, damit sich die eingestreuten Störsignale im ideall Fall substrahieren. Weiterhin soll die im Sensor eingesetzte Elektronik rauscharm sein und am besten steurbar sein, um einen besseren Signal-Rausch-Verhältnis zu erzielen.

Ein zweiter wesentlicher Aspekt beim Entwurf des EKGSensors ist der Energieverbrauch. Da der EKG-Sensor tragbar ist, sollte seine Batterie nicht sehr beansprucht werden und für mehrere Tage halten. Der Leistungsverbrauch der EKG-Einheit kann in drei Teile unterteilt werden: eine Messleistung für die Ableitung des Signals, eine Sendeleistung, und eine Rechenleistung für die Signalauswertung.

Eine geringe Messleistung erfordert einen niedrigen Stromverbrauch der elektronischen Schaltungen, ein $\mathrm{Ab}$ schalten der Elektronik falls keine Messung vorhanden ist, und eine niedrige Abtastrate des Messsignals, die mit einem Anpassen an dem Messbereich erfüllt sein kann. Die Sendeleistung kann reduziert werden, indem nur Nutzdaten des Signals gesendet werden, und ein energiesparendes Auswerteverfahren (Ayari-Tielert, 2005) kann anschließend die Rechenleistung verringern. Das Verfahren soll auf das Messsignal adaptiv reagieren.

\subsection{System-Architektur}

Das in dieser Arbeit entwickelte System hat folgende Architektur, siehe Abb. 2. Es besteht aus einem EKG-Sensor, einer Funkstelle und einer Monitoring Einheit.

Der EKG-Sensor ist ein 1-Kanal-Sensor und basiert auf einer bipolaren Ableitung, bei dem durch einen Brustgurt zwei Elektroden an der Brust angebracht werden. Mit den EKG-Elektroden werden Potentialdifferenzen zwischen zwei Punkten auf der Körperoberfläche erfasst, die sich aufgrund dieser Potentialverteilung ergeben. Das Messsignal wird am Eingang mit einer konstanten niedrigen Grenzfrequenz zur Unterdrückung von den Hautkontaktpotenzialen Hochpass gefiltert, zuerst mit einem Instrumentenverstärker fester Verstärkung zu einem im 100 mV-Bereich Signal, dann mit Hilfe einer steuerbaren Front End Schaltung, siehe Abschnitt 4, variabel zu einem im V-Bereich Signal verstärkt, und anschließend nach seinen hochfrequenten Störsignalen variabel Tiefpass gefiltert. Die Einstellung der variablen 


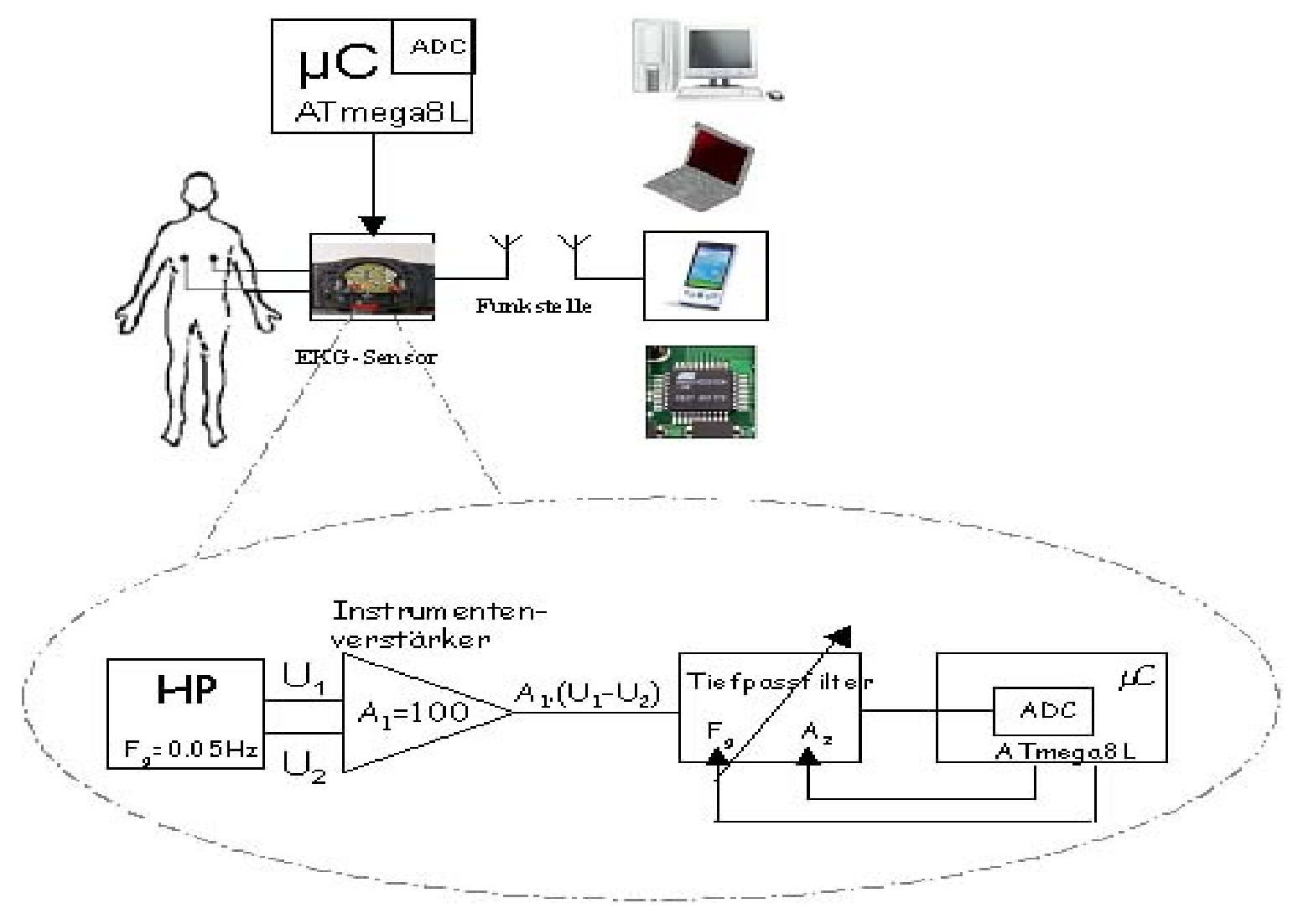

Abb. 2. System-Architektur.

Verstärkung und Grenzfrequenz geschieht durch einen Mikrocontroller, den ATmega8L, der einen 10-Bit A/D-Wandler enthält, mit dem eine adaptive Abtastung des Messsignals erfolgt. Die Funkübertragung hat den Vorteil die CommonMode Störungen zu unterdrücken und eine aussagekräftigere Signalauswertung dank der Beseitigung der Artefakten zu ermöglichen, die durch die Bewegung der Elektrodenkabel bei einer stationären Messung mit vollständiger Verkabelung des Patienten bedingt wäre.

Die Funkstelle ist vorläufig durch ein kommerzielles System von der Firma Adcon RF-technology realisiert worden und wird durch eine Nah-Feld-Übertragung ersetzt.

Die Monitoring Einheit kann je nach Anwendung ein Rechner, ein Laptop, ein PDA oder sogar ein 8-Bit Mikroprozessor sein. Dort laufen ein interaktiver Auswertealgorithmus und die visuelle Datenausgabe.

\section{Adaptiver Switched-Capacitor Filter}

Die in Abschnitt 3.2 beschriebene steuerbare Front End Schaltung ist als ein adaptiver Switched-Capacitor Filter realisiert. Der Filter ist ein Butterworth Tiefpass zweiter Ordnung. Die Schaltung ist in Abb. 3 dargestellt.

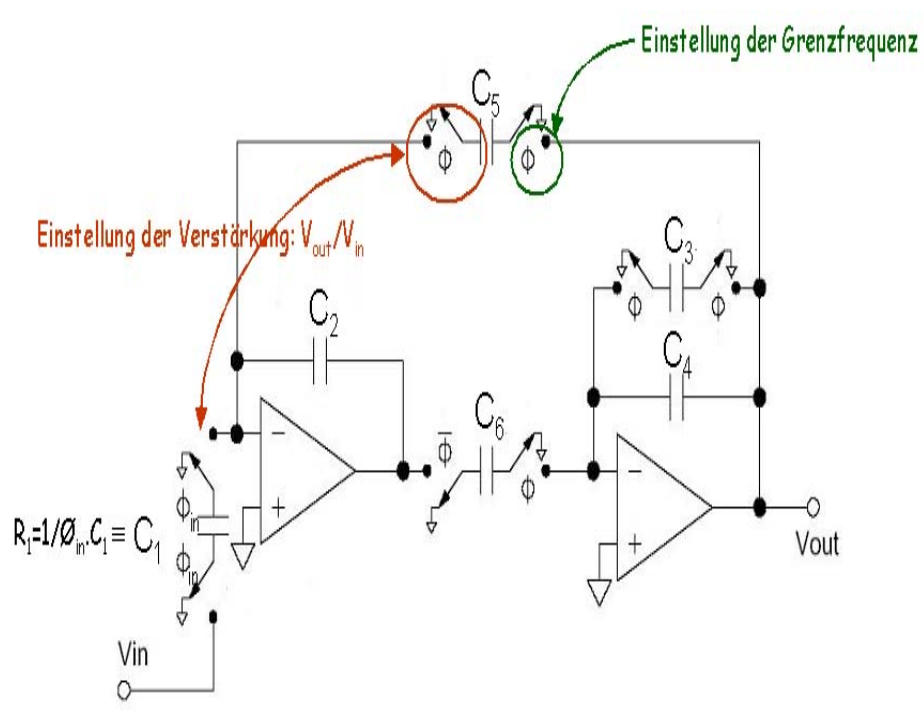

Abb. 3. Switched-Capacitor Filter mit variabler Verstärkung. 

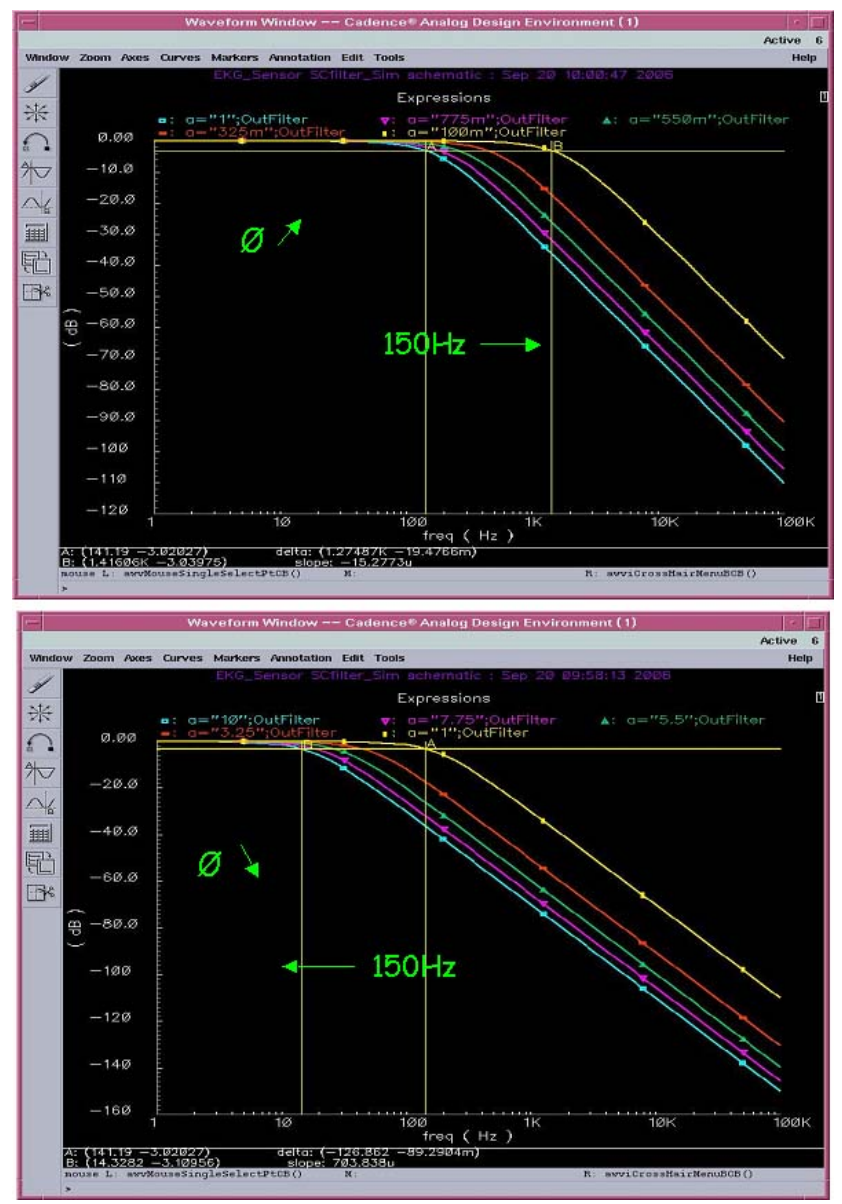

Abb. 4. Variation der Grenzfrequenz Fg.

Die Schaltung ist durch zwei Taktfrequenzen $\phi$ und $\phi_{i n}$ gekennzeichnet. Die Haupttaktfrequenz $\phi$ ist für die Einstellung der Grenzfrequenz des Filters verantwortlich. Wird $\phi$ vergrössert, erhöht sich die Grenzfrequenz und umgekehrt. In Abb. 4 sind mehrere Amplitudengänge des Filters in Abhängigkeit von $\phi$ aufgetragen. Dabei ist $\phi_{i n}=\phi=2 \mathrm{kHz}$. Für eine Grenzfrequenz von $150 \mathrm{~Hz}$ hat sie den Wert von $2 \mathrm{kHz}$. Dieser Wert ist ein Optimum zwischen einem minimalen Rauschen der Kapazitäten in der Schaltung und einer minimalen Fläche dieser Kapazitäten.

Das Verhältnis zwischen der Haupttaktfrequenz $\phi$ und der Eingangstaktfrequenz $\phi_{i n}$ ist für die Variation der Filterverstärkung maßgebend. Dieses Verhältnis ist umgekehrt proportional zu der Verstärkung A, siehe Abb. 5. Hier ist der Wert von $\phi$ konstant gehalten auf den Wert von $2 \mathrm{kHz}$ und die Eingangstaktfrequenz $\phi_{i n}$ variiert. Für die gleiche Grenzfrequenz von $150 \mathrm{~Hz}$ und für eine Schaltungsverstärkung von 10 hat $\phi_{\text {in }}$ folglich den Wert von $20 \mathrm{kHz}$.

Der adaptive Switched-Capacitor Filter wurde in einem $0.18 \mu \mathrm{m}$ CMOS-Prozess realisiert. Er ist mit einer

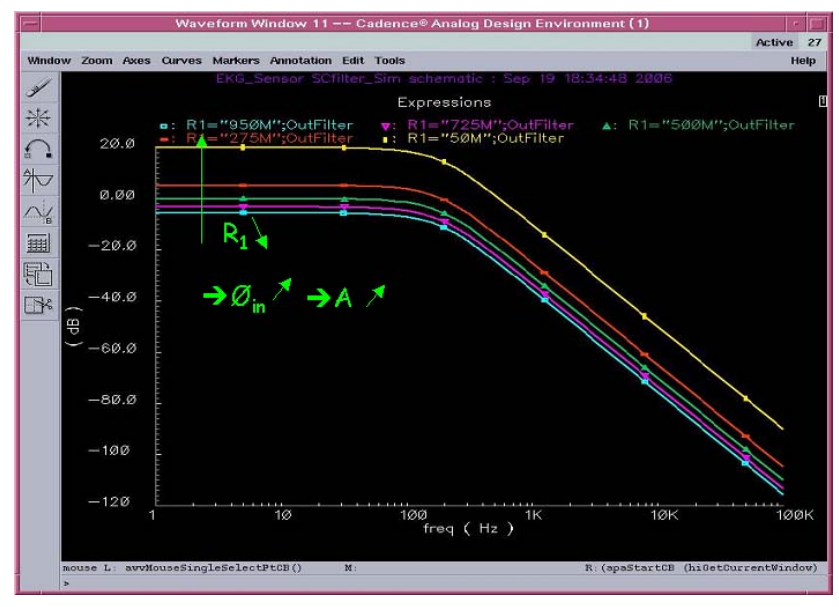

Abb. 5. Variation der Verstärkung A.

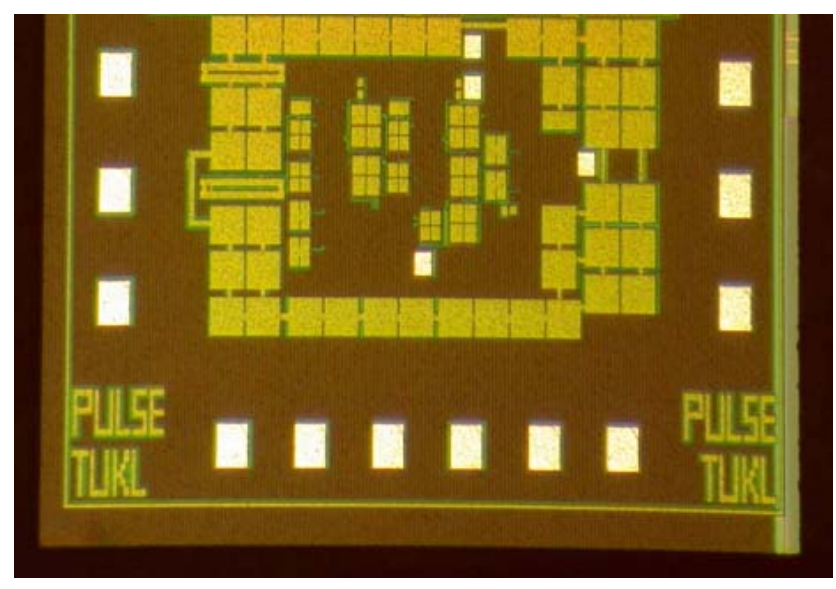

Abb. 6. Chipfoto.

Versorgungsspannung von $3 \mathrm{~V}$ betrieben und hat einen Energieverbrauch von $600 \mu \mathrm{W}$. Seine aktive Fläche ist $420 \mu \mathrm{m} \times 320 \mu \mathrm{m}$. Abbildung 6 zeigt das Chipfoto.

Die Variabilität der Filtergrenzfrequenz und seiner Verstärkung bietet den Vorteil, einerseits die Signalbandbreite einregulieren zu können, je nachdem, welche Welle des EKG-Signals ausgewertet wird und damit mehr Rauschen filtern zu können, und anderseits den Aussteuerungsbereich am Eingang des A/D-Wandlers zu verbessern.

\section{Drahtlose online Auswertung}

Zwei Methoden zur Online Auswertung werden in dieser Arbeit vorgestellt. Je nach Anwendung und Anforderungen an die Signalextraktion wird zwischen den beiden Methoden gewählt. Bei der ersten Methode läuft der 
Auswertealgorithmus auf einem funkgebundenen Auswertungsrechner, der ein PC, ein Laptop, ein PDA oder ein 8-Bit Mikroprozessor sein kann. In diesem Fall werden die abgetasteten Signalwerte über die Funkstelle zum Auswertungsrechner übertragen, und der Least Squares Fit mit tabellierten Funktionen, siehe Abschnitt 5.4, wird als Auswertealgorithmus eingesetzt.

Bei der zweiten Methode läuft der Auswertealgorithmus lokal auf dem Mikrocontroller, der unter anderem die Front End Schaltung steuert. Hier beruht der eingesetzte Algorithmus auf Korrelation, siehe Abschnitt 5.5, und es werden nur die aus dem Messsignal extrahierten Parameter zu der Monitoring Einheit gesendet.

Beide Methoden benutzen das gleiche energiesparende Verfahren, das EKG-Signal auszuwerten. Es wird auf dieses Verfahren in Abschnitt 5.2 eingegangen. Dieses Verfahren ist durch eine Interaktion zwischen der messenden Elektronik und dem Auswerteprogramm bezeichnet, bei der der Auswertealgorithmus eine adaptive Abtastung kontrolliert, in dem er Anfang und Ende der Messintervalle des A/DWandlers und seine Abtastfrequenz bestimmt. Das Verfahren ist ausserdem durch die Anwendung von Musterschablonen charakterisiert. Sie beinhalten die grundsetzlichen Signalinformationen und erlauben somit eine Unterabtastung des Signals, siehe Abschnitt 5.2.

\subsection{Musterschablonen des EKG-Signals}

Aus dem Elektrokardiogramm werden drei Musterschablonen extrahiert. Sie sind in Abb. 7 dargestellt. Eine Schablone für die P-, T-, und U-Wellen, eine zweite für den QRSKomplex und eine dritte für das ST-Segment. Diese Schablonen beinhalten Kurveninformationen der verschiedenen Signalabschnitte und bestehen aus klassifizierten Daten je nach Signalbereich. Sie können in einem ersten Schritt aus einer typischen EKG-Kurve, oder spezifisch aus dem EKG jedes zu untersuchenden Patienten in einer Kalibrierungsphase des EKG-Sensors entnommen werden.

Jede Schablone ist allgemein mit einem Tupel (H: Höhe, B: Breite, V: Verschiebung in t, Off: Offset) beschreibbar. Diese Parameter charkterisieren den Signalabschnitt mit der entsprechenden Musterschablone auf der Zeit- und Amplituden-Achsen.

\subsection{Energiesparendes Verfahren zur EKG-Auswertung}

Die Auswertung des Elektrokardiogramms besteht aus zwei Phasen, siehe Abb. 8. In einer ersten Phase wird das Signal während einem beschränkten Zeitintervall kontinuierlich mit einer festen Abtastfrequenz grob abgetastet. Die Daten werden analysiert und Heuristiken werden zur Schätzung der Parameter $\left(H_{i}, \mathrm{Off}_{i}, B_{i}, V_{i}\right)$ der verschiedenen Wellen durchgeführt. Die Signal-Nulllinie wird aus einer Mittelwertbildung der erhaltenen Daten einer Periode ermittelt. Ihre Höhe kann als abgeschätzter Wert des Wellenoffsets

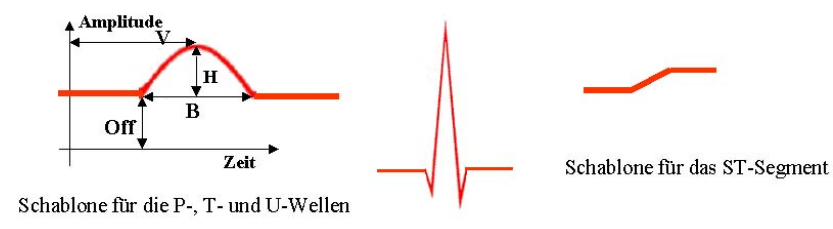

Schablone für den QRS-Komplex

Abb. 7. EKG-Musterschablonen.

betrachtet werden. Des weiteren werden die Höhen- und Verschiebungs-Parameter durch eine Maximumsuche in jedem Signalabschnitt berechnet. Die Wellenbreiten werden aus den Kurvenänderungen hergeleitet.

Die zweite Phase ist durch ein prädiktives Verfahren gekennzeichnet und die Abtastung verläuft nur in relevanten Bereichen des Signals. Die abgeschätzten Parameter in der ersten Phase dienen zur Festlegung der Signalfenster. Die Ergebnisse der gefitteten Wellen werden als Anfangswerte der Parameter der nächsten Periode benutzt, so dass eine laufende Korrektur der Lokalisierung der Messfenster im Signal stattfindet. Bei falscher Konvergenz des Algorithmus werden die Ergebnisse des Algorithmus verworfen und eine neue Heuristik zur Abschätzung der Wellenparameter durchgeführt.

\subsection{Berechnung der EKG-Parameter}

Aus den Wellenparametern, die mit dem Fitting Algorithmus ermittelt werden, werden die EKG-Parameter berechnet.

1. $R-$ Zacke $=\left[H_{\mathrm{QRS}}(i) * H_{\mathrm{NQRS}}\right]+\operatorname{Off}_{\mathrm{QRS}}(i)$

2. $R-R-\operatorname{Zeit}[\mathrm{ms}]=V_{\mathrm{QRS}}(i+1)-V_{\mathrm{QRS}}(i)$

3. Herzfrequenz:Herzschläge/Minute $=60 * 1000 / V_{\mathrm{QRS}}(i+1)$ $-V_{\mathrm{QRS}}(i)[\mathrm{ms}]$

4. $P$ - Amplitude $=\left[H_{P}(i) * H_{N P}\right]+\operatorname{Off}_{P}(i)$

5. $P$ - Dauer $=B_{P} * B_{N} P$

6. $P Q-\mathrm{Zei} t=V_{\mathrm{QRS}} * V_{P}(i)$

7. $\mathrm{QRS}$-Intervall $=B_{\mathrm{QRS}} * B_{\mathrm{NQRS}}$

8. $Q T-$ Zeit $=\left[V_{T}(i) * V_{\mathrm{QRS}}(i)\right]+B_{T}(i) * B_{N T}$

9. $S T-$ Strecke $=\left[V_{\mathrm{QRS}}(i)+B_{\mathrm{QRS}}(i) * B_{\mathrm{NQRS}}\right]-V_{T}(i)$

10. $T$ - Amplitude $=\left[H_{T}(i) * H_{N T}\right]+\operatorname{Off}_{T}(i)$ 
1. Phase

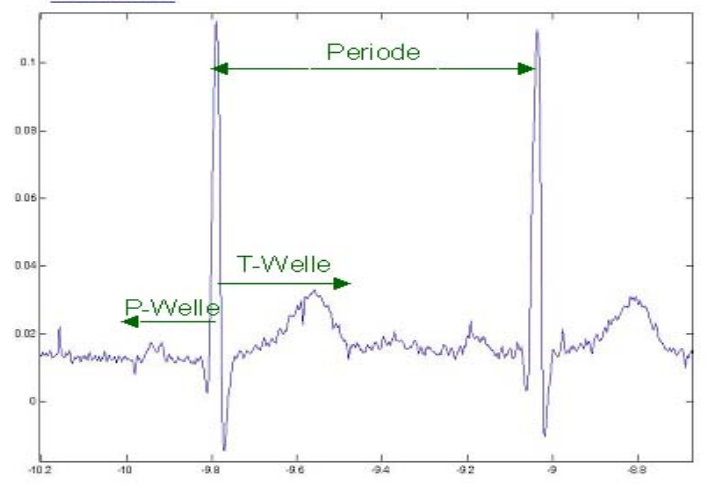

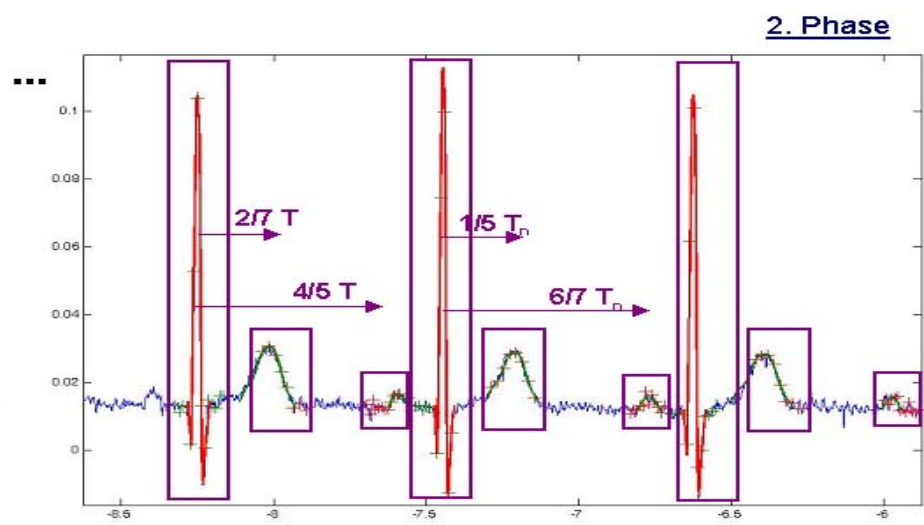

Abb. 8. EKG-Auswertung.

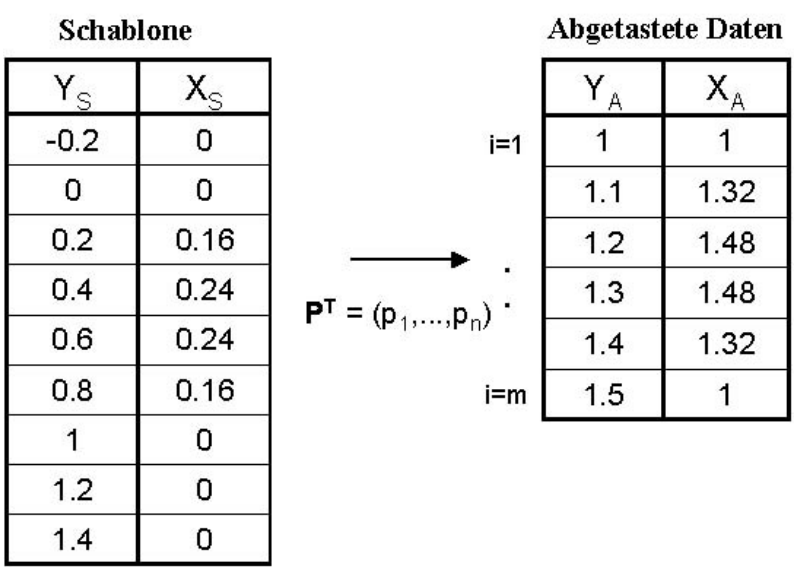

Abb. 9. Beispiel fur eine Transformation der Schablone bei Fit.

\subsection{Least Squares Fit mit tabellierten Funktionen}

Eine Methode mit guter Konvergenz zur nichtlinearen Ausgleichrechnung, der Levenberg-Marquardt Algorithmus (Marquardt, 1963) ist bereits vielfach eingesetzt. Dieser Algorithmus kann zum Anpassen von Parametern, die die nichtlineare Modellkurve beschreiben, genutzt werden (Bevington, 1969).

In vielen Fällen ist es aber schwierig, eine Zielfunktion als Modellkurve für die erhaltenen Messdaten analytisch zu erstellen. In den meisten Fällen ist die nichtlineare Zielfunktion entweder zu komplex, oder gar nicht erstellbar und wird durch Approximationen ersetzt, die zusätzliche Fehler in der Ausgleichsrechnung beziehen.

Eine Modifizierung in der Anwendung der LevenbergMarquardt Methode zur Umgehung dieses Problems besteht darin, auf eine analytische Zielfunktion zu verzichten und sie durch tabellierte Kurven zu ersetzen.

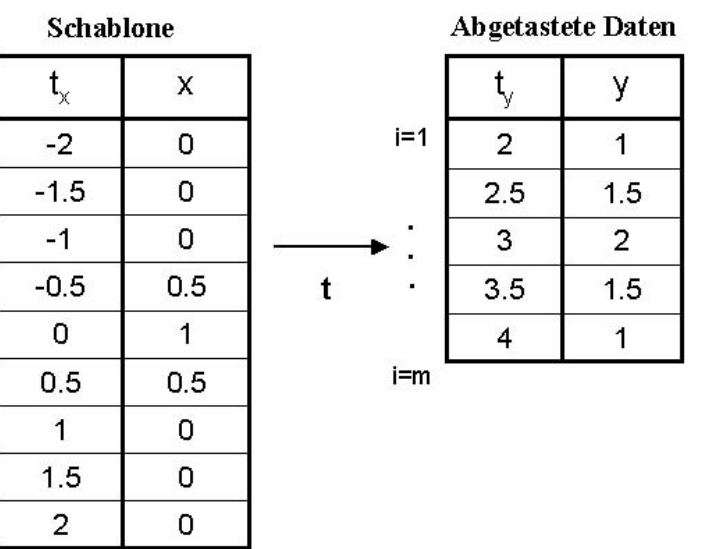

Abb. 10. Beispiel fur eine Transformation der Schablone bei Korrelation.

Ein standardisiertes Signal wird als Schablone definiert und in diskreter Form tabelliert. Ein Beispiel dazu sieht man in Abb. 9. Die Schablone wird durch die Transformation $P^{T}$ mit n Parametern an die abgetasteten Daten angenähert. In dem Fall der EKG-Auswertung werden die Parameter Höhe, Breite, Verschiebung in $t$, und Offset verwendet.

Die im Algorithmus benötigten Ableitungen der Zielfunktion nach den Parametern werden numerisch gebildet. Es ist wichtig den Ableitungsschritt zu optimieren, damit keine Rechenfehler eintreten.

\subsection{Korrelation}

In dieser Arbeit wird die Korrelation eingesetzt, um die RZacke des EKG-Signals auf der Zeit-Achse zu lokalisieren, und folglich den RR-Abstand zwischen zwei nacheinander folgenden R-Zacken zu berechnen. Dafür wird die QRS-Schablone auf der Zeit-Achse verschoben und mit den 

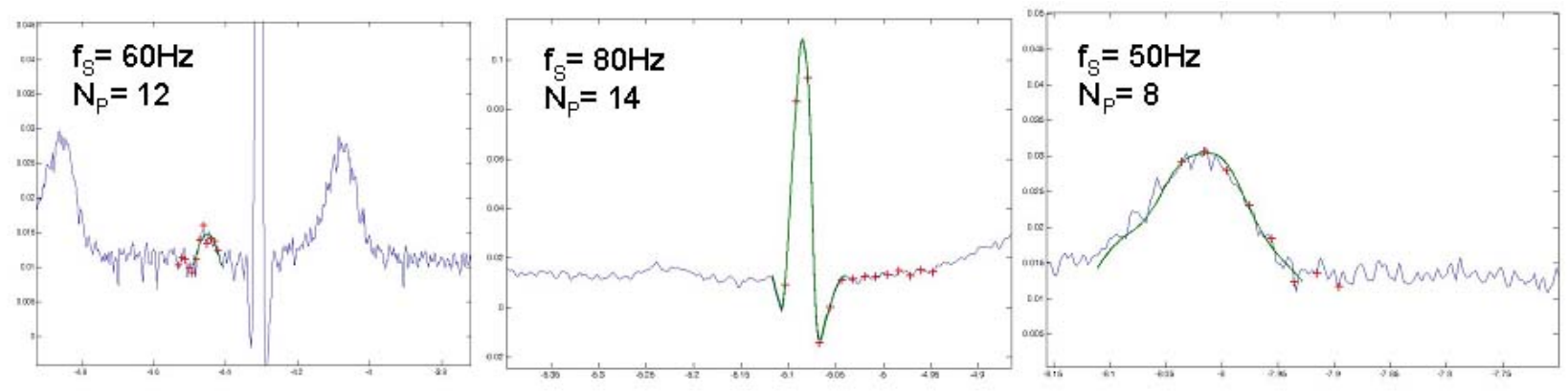

Abb. 11. Gefittete Wellen in dem gemessenen EKG-Signal.

abgetastetenv Daten korreliert, bis der Korrelationskoeffizient seinen maximalen Wert erreicht, siehe Abb. 10. Da es aber von Energievorteil so wenig wie möglich abzutasten, hat die Daten-Reihe in der Regel weniger Elemente als die Schablone. Deswegen wird im Algorithmus aus dem Verschiebungsparameter und der QRS-Schablone eine transformierte Schablone errechnet, die die gleiche Anzahl der Elemente wie die Daten-Reihe besitzt, und die mit den abgetasteten Daten korreliert wird. Der Algorithmus wird durch einen Schätzwert der Verschiebung beschleunigt.

\section{Ergebnisse}

In Abb. 11 werden die aus den Abtastwerten (rote Kreuze) gefitteten Kurven (grüne Kurven) mit dem abgeleiteten Signal (blaue Kurve) zum Vergleich dargestellt. Die Abb. 11 zeigt, dass die gefitteten Wellen und die gemessenen Wellen sehr gut übereinander liegen. $f_{S}$ und $N_{P}$ stehen jeweils für die Abtastfrequenz und die Anzahl der Abtastpunkte.

Im Beispiel der Abb. 11 wurden unterschiedliche Abtastfrequenzen von $50 \mathrm{~Hz}$ für die T-welle, von $60 \mathrm{~Hz}$ für die Pwelle und von $80 \mathrm{~Hz}$ für das QRS-Komplex eingestellt, um das EKG-Signal zu charakterisieren.

Eine Abtastfrequenz von nur $80 \mathrm{~Hz}$ reicht aus, um den QRS-Komplex sehr gut zu rekonstruieren. Unterabtastung ist erlaubt, da die verwendeten Schablonen die grundsetzlichen Signalinformationen schon beinhalten. Als Konsequenz sind pro Periode nur etwa 34 Abtaspunkte nötig, um die Parameter der P-, T-Wellen und des QRS-Komplexes zu extrahieren.

Nicht zuletzt wird das in den Messdaten enthaltene Rauschen durch das Fitting reduziert.

Abbildung 12 zeigt die Extraktion der R-ZackenVerschiebung mit Hilfe von Korrelation. Diese extrahierten Werte weichen um circa $0.5 \mathrm{~ms}$ von den von Hand ermittelten Werten $V_{1}($ Hand $)=4.304 \mathrm{~s}, V_{2}$ (Hand) $=5.086 \mathrm{~s} \mathrm{ab}$.

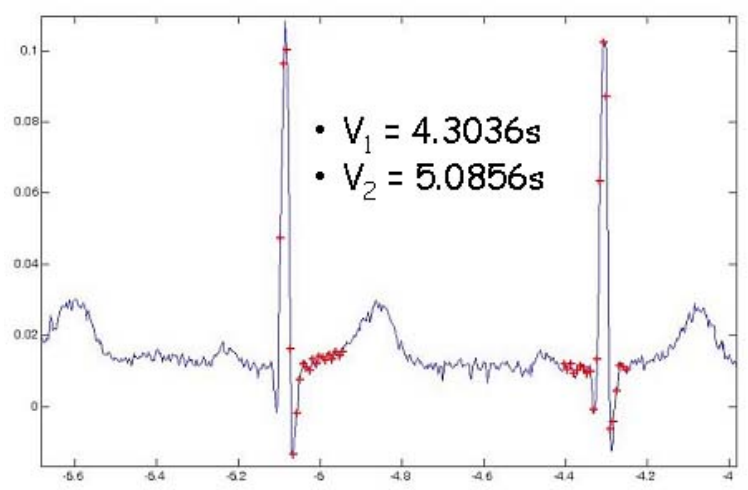

Abb. 12. Extrahierte Verschiebung der R-Zacke mit Korrelation.

\section{Zusammenfassung}

Ein mobiles EKG-System zur Online Auswertung von EKGSignalen wurde vorgestellt. Das System setzt sich zusammen aus einem EKG-Sensor zur Erfassung des Signals, einer Funkstelle und einer Monitoring Einheit, die auch den Auswertungsrechner enthält. Der EKG-Sensor besteht aus einem Hochpass, einem Instrumentenverstärker, einer durch einen Mikrocontroller adaptiv steuerbaren Front End Schaltung, die ihre Verstärkung und Grenzfrequenz einstellen lässt, und einem im Mikrocontroller integriertem A/D-Wandler, der die adaptive Abtastung des Signals übernimmt. Die Variabilität der Filtergrenzfrequenz hat den Vorteil mehr Rauschen vom Nutzsignal beseitigen zu können, und die seiner Verstärkung den Vorteil den vollen Austeuerungsbereich des A/D-Wandlers auszunutzen, und folglich die Auflösungsgenauigkeit des Signals zu erhöhen. Die Front End Schaltung wurde in einem $0.18 \mu \mathrm{m}$ CMOS-Prozess realisiert. Sie ist mit einer Versorgungsspannung von $3 \mathrm{~V}$ betrieben und hat einen Energieverbrauch von $600 \mu \mathrm{W}$. Ihre aktive Fläche ist $420 \mu \mathrm{m} \times 320 \mu \mathrm{m}$. Die Online Auswertung des EKG-Signals arbeitet je nach Anforderungen an die Signalextraktion mit zwei Algorithmen, das Least Squares Fit mit tabellierten Funktionen und die Korrelation. 


\section{Literatur}

Ayari, E. and Tielert, R.: Verfahren für eine kontinuierliche drahtlose EKG-Auswertung, Kleinheubacher Tagung 2005, Miltenberg, Sept. 2005.

Wikipedia: Elektrokardiogramm, http://de.wikipedia.org/wiki/ Elektrokardiogramm.
Meyer-Waarden, K.: Bioelektrische Signale und ihre Ableitverfahren, Schattauer, März 1984.

Marquardt, D.: An Algorithm for Least-Squares Estimation of Nonlinear Parameters, J. Soc. Appl. Math. 11, p. 431, 1963.

Bevington, P.: Data reduction and error analysis for the physical sciences, New York: McGraw-Hill, 1969. 